\title{
¿INCLUSIVIDAD EN LAS HERRAMIENTAS WEB 2.0?*
}

\author{
Verónica Marín-Díaz ${ }^{1}$ \\ Begoña Esther SAMPedro-Requena ${ }^{1}$ \\ Jorge Francisco Figueroa Flores ${ }^{2}$
}

\begin{abstract}
RESUMEN: Los procesos educativos inclusivos y las TIC son dos de los elementos considerados relevantes. En estas líneas presentamos los principales resultados obtenidos en una investigación relativa a determinar cuál es la valoración que los estudiantes de las extintas titulaciones de Maestro de la Universidad de Córdoba le otorgan a diversas herramientas 2.0 en los entornos inclusivos. Se ha empleado un diseño no experimental y descriptivo, utilizando un cuestionario tipo Likert, para la recogida de los datos. Los principales resultados indican que el uso de estos depende de la especialidad cursada. También, se ha encontrado que los edublogs no son valoradas como elementos inclusivos. Por ello se puede concluir que es necesaria una formación inclusiva de los futuros maestros
\end{abstract}

Palabras clave: Web 2.0. Educación inclusiva. formación inicial.

\section{QUESTIONING INCLUSIVE ENVIRONMENTS IN THE ACADEMIC USE OF THE WEB 2.0 TOOLS}

\begin{abstract}
Nowadays, two of the most relevant topics addressed by the educational community are Inclusive Education (IE) and Information and Communication Technologies (ICT's). The following paper presents the main results obtained in a recent research conducted at Universidad de Córdoba. It focused on determining the level of importance undergraduate college students, majoring in education, give to Web 2.0 tools in inclusive environments. The research was conducted following a non-experimental and descriptive research design, using the Likert scale questionnaire for data collection. The main results indicate that such use depends on their course of graduation. In addition, it was found that educational tools such as "edublogs" are not seen as inclusive elements. By this means, it is necessary to include IE training in the curriculum of future educators.
\end{abstract}

Keywords: Web 2.0. Inclusive education. Teacher education.

\footnotetext{
*Proyecto Aprender a través de la formación en competencias II (2013-R-11-6004), concedido por la Universidad de Córdoba (España), curso académico 2014-2015.

${ }^{1}$ Universidad de Córdoba, Facultad de Ciencias de la Educación - Córdoba, España.

E-mails: vmarin@uco.es, bsampedro@uco.es

${ }^{2}$ Universidad del Este de Puerto Rico, Escuela de Ciencias Sociales y Humanas - Carolina, Puerto Rico.

E-mail: jffigueroa@suagm.edu
}

DOI: 10.1590/ES0101-73302018164908 


\section{EN METTANT EN QUESTION LES ENVIRONNEMENTS INCLUSIFS DANS L'USAGE ACADÉMIQUE DES OUTILS WEB 2.0}

RESUME: Processus éducatifs inclusifs et information et communication technologies sont, à l'heure actuelle, deux des affaires que la communauté éducative considère les plus importants. Dans ces lignes, nous présentons les principaux résultats d'une enquête qui s'est tenue à l'Université de Cordoba, en concernant de déterminer l'importance que les étudiants du domaine éducatif attachent aux outils Web 2.0 dans les environnements inclusifs. On a utilisé une conception non expérimentale et descriptive, à l'aide d'échelle de Likert pour la collecte de données. Les principaux résultats tournent autour de différents usages que les étudiants donnent aux outils Web 2.0, qui sont en particulier different selon les objectifs du cours. Il a aussi été établi que les outils pédagogiques appropriés comme Edublogs ne sont pas vus comme éléments d'inclusion. Par conséquent, on peut conclure que la formation inclusive des futurs enseignants est nécessaire.

Mots-clés: Web 2.0. Éducation inclusive. Formation initiale.

\section{Introducción}

L

a atención a la diversidad, concretamente los contextos y procesos educativos inclusivos y la tecnología educativa, particularmente las herramientas Web 2.0, son dos aspectos enormemente amplios de la educación y, en los cuales se sustenta el presente estudio. Estas dos temáticas son consideradas el eje vertebrador de los nuevos modelos educativos que plantearan nuevas metodologías didácticas en las aulas de cualquier nivel educativo, motivo por lo que se aplican cada vez con más asiduidad, en el ámbito de la formación inicial del docente (MARÍN, 2016).

Cuando hablamos de formación inicial nos referimos al proceso de aprendizaje que realizan los estudiantes durante sus estudios universitarios, en este caso, los conducentes al título de maestro.

"La educación superior debe intensificar la formación docente con currículas que proporcionen los conocimientos y las herramientas necesarios para el siglo XXI. Esto requerirá nuevos abordajes, que incluyan la educación abierta y a distancia (EAD) e incorporen tecnologías de la información y la comunicación" (UNESCO, 2009, p. 5).

En este sentido la UNESCO en 2008 ya señalaba que "los programas de formación inicial para futuros profesores deben comprender en todos los elemen- 
tos de la capacitación experiencias enriquecedoras con TIC" (UNESCO, 2008b, p. 1). En consecuencia, estas han de ser consideradas como el desencadenante de un cambio de funciones, rol y formas de afrontar la acción docente si queremos su integración en la educación, tal y como indica Castaño et al. (2008), y la UNESCO (2008a, p. 7), al afirmar que "en el futuro, las competencias fundamentales comprenderán la capacidad tanto para desarrollar métodos innovadores de utilización de TIC en el mejoramiento del entorno de aprendizaje, como para estimular la adquisición de nociones básicas en TIC, profundizar el conocimiento y generarlo".

Por tanto, podemos entender que los paradigmas educativos y las prácticas y acciones que de ellos se derivan, están íntimamente relacionados con el desempeño de la labor docente, por esta razón una formación inicial que esté acorde con las exigencias y expectativas de la educación actual, considerando dentro, la formación y capacitación para incorporar a las TIC en los procesos de enseñanzaaprendizaje, favorecerá una educación de calidad.

Los estudios realizados por Boza y Conde (2015), Grieve, Padgett y Moffitt (2015) sobre las creencias que subyacen en el uso de las TIC en los estudiantes de Magisterio, en relación a su formación inicial, pone de relieve lo que ya indicaba el estudio de la UNESCO (2008a), anteriormente señalado, el cual concluye que los alumnos consideran que las TIC pueden mejorará la acción docente pero para ello se necesita tener capacidad innovadora, por lo que necesitaran el fortalecimiento de su propia autonomía dentro de las clases, con el fin de propiciar espacios que desarrollen todas sus capacidades, por otra parte también consideran despiertan interés.

Bajo este prisma, se entiende que los escenarios educativos de la etapa de Educación Primaria, están en continuo cambio, derivando estas transformaciones, en su mayoría, de los nuevos recursos tecnológicos que se van produciendo de forma vertiginosa.

En consecuencia, si "el movimiento de la inclusión persigue la construcción de una nueva escuela en la que las diferencias individuales se contemplen como un valor y no como un problema y donde todos los miembros conformen una auténtica comunidad educativa con un sentido acuciado de pertenencia" (GALLEGO; RODRÍGUEZ, 2007, p. 108), es lógico pensar que la formación de los formadores deben contar con esta perspectiva desarrollada bajo el paraguas de las TIC, dada la importante presencia que estas últimas tienen en las vidas de los individuos. El empleo de estas para el desarrollo de una educación inclusiva, va a permitir que los sujetos puedan interactuar con otros de forma activa.

Es por ello, que debemos considerar los aspectos positivos, alejándonos de los inconvenientes que pueda generar la vinculación inclusión-TIC, dado que "el principal aspecto positivo de la relación en TIC-educación inclusiva es el poder crear tanto estrategias de enseñanza como procesos de aprendizaje" (MARÍN, 2013, p. 99). 
Compartimos con Marín (2013) y Carrasco, Baldivieso y Di Lorenzo (2016) que el desarrollo de las TIC en general y de Internet en particular, han sido una conmoción para la vida diaria de los sujetos, es por ello que estas han ido determinando a su vez el desarrollo de una educación para la inclusión de los sujetos en el devenir de la sociedad. No obstante la evolución de las TIC merece una mención especial en este documento, dado que las entendemos o queremos entenderlas como moduladoras de los currículos inclusivos que los futuros docentes del mañana vayan desarrollar.

Las tecnologías de la información y la comunicación en educación: La Web 2.0

Actualmente estamos asistiendo a una gran transformación en el terreno de las TIC, las denominadas herramientas Web 2.0, las cuales están mejorando de manera sustancial los procesos educativos y con ellos el desarrollo de la educación, en los centros escolares, tal como indican autores como Boza y Conde (2015), Roblizo y Cozar (2015) y Cozar-Gutiérrez y Sáez-López (2017).

La innovación permanente en la acción y en la práctica del docente, en todos los niveles educativos pero sin duda en la etapa de primaria, es necesaria e ineludible como ha quedado patente hasta ahora, con más fuerza si cabe, tal y como revela Araujo (2016). Por esta razón, no debemos perder de vista que las TIC siguen avanzando en el tiempo, dando lugar a nuevos contextos de educación, y, por tanto, a nuevas prácticas educativas y acciones pedagógicas. Prueba de ello lo encontramos en las investigaciones y experiencias que en torno a ella se han llevado en los últimos tiempos (ARAUJO, 2016; DABBAGH; FAKE, 2017), en los que se pone de manifiesto la gran penetración que estos recursos están teniendo en los procesos de enseñanza y aprendizaje.

Los elementos o rasgos que definen a las TIC han variado, pero sin duda el atributo más relevante de esta nueva Web es su carácter más accesible, es decir, como apunta Castańo et al. (2008), ya no es necesario tener unos conocimientos específicos en informática o sistemas o en gestión de contenidos, sino simplemente conocimientos elementales para poder abordar el uso práctico de las TIC. Por lo que el uso y empleo de las herramientas tecnológicas en la educación varía la forma de entender y contemplar a la misma, entendiendo que las tecnologías pueden ayudarnos a cambiar el proceso de enseñanza - aprendizaje, haciéndolo más flexible, dinámico, motivador y accesible para todos los implicados (BOZA; CONDE, 2015).

Castaño et al. (2008) apunta a este respecto, que las diferentes prácticas educativas que se llevan a cabo en un entorno educativo concreto son, ahora más que nunca, conocidas por el resto de la comunidad docente, favoreciendo de esta forma las adaptaciones de las innovaciones pedagógicas en los diferentes espacios educativos. La literatura científica sobre Web 2.0 y las herramientas que la desarrollan es muy extensa, como ya hemos indicado (ARAUJO, 2016; BOZA; 
CONDE, 2015; DE LA HORRA, 2017; MARIÑO; ALFONZO; CAVALIERI, 2014; MIRABAL; GÓMEZ; GONZÁLEZ, 2015). Entre las distintas clasificaciones que existen se encuentra la que realizan Roblizo y Cozar (2015), la cual se apoya en la de Mcgee y Díaz (2007 apud CABERO, 2009, p. 21), algunas de las cuales se exponen en la Tabla 1 considerando su función y tipo.

Por otra parte, Castaño et al. (2008) realiza otra categorización basada en la aplicabilidad de las mismas y tomando como referencia la de Mcgee y Díaz. Nos decantamos por el uso de la de Castaño et al. (2008) considerar que abraca todos los espacios en los que la red se expande: las aplicaciones online (en las que se incluirían los procesadores de texto, las hojas de cálculo, las presentaciones, los calendarios, las bases de datos, etc.), las herramientas de publicación (Webquest, wiki, blogs y su versión educativa edublogs) y la gestión de información (sindicadores de contenido, páginas de inicio, marcadores sociales).

\section{Tabla 1}

Clasificación de algunas herramientas Web 2.0 atendiendo a su tipo y función.

\begin{tabular}{|c|c|c|}
\hline Tipo & Función & Herramientas \\
\hline De comunicación & $\begin{array}{l}\text { Para compartir ideas e } \\
\text { información }\end{array}$ & $\begin{array}{l}\text { - Blogs } \\
\text { - Audioblogs } \\
\text { - Videoblogs } \\
\text { - Mensajería instantánea } \\
\text { - Podcats } \\
\text { - Webcams }\end{array}$ \\
\hline De colaboración & $\begin{array}{l}\text { Para trabajar con otras } \\
\text { personas, por un objetivo } \\
\text { específico, en un espacio de } \\
\text { trabajo compartido }\end{array}$ & $\begin{array}{l}\text { - De edición y escritura } \\
\text { - Comunicaciones virtuales de prácticas } \\
\text { - Wikis }\end{array}$ \\
\hline De documentación & $\begin{array}{l}\text { Para recolectar o presentar } \\
\text { evidencias de experiencias, } \\
\text { producciones, líneas de } \\
\text { pensamiento en el tiempo, etc. }\end{array}$ & $\begin{array}{l}\text { - Blogs } \\
\text { - Videoblogs } \\
\text { - Portafolios electrónico }\end{array}$ \\
\hline De creación & $\begin{array}{l}\text { Para crear algo nuevo que } \\
\text { puede ser visto y/o usado por } \\
\text { otros }\end{array}$ & $\begin{array}{l}\text { - Aplicaciones Web hibridas } \\
\text { - Comunidades virtuales de practicas } \\
\text { - Mundos virtuales de aprendizaje }\end{array}$ \\
\hline De interacción & $\begin{array}{l}\text { Para intercambio de } \\
\text { información, ideas, recursos, } \\
\text { materiales }\end{array}$ & $\begin{array}{l}\text { - Objetos de aprendizaje } \\
\text { - Marcadores sociales } \\
\text { - Comunidades virtuales de practicas } \\
\text { - Mundos virtuales de aprendizaje }\end{array}$ \\
\hline
\end{tabular}

Fuente: Cabero (2009, p. 21). 


\section{Objetivos, metodología y diseño}

La investigación planteada presenta como objetivo general conocer la valoración que tienen los futuros maestros en formación, de la etapa de educación primaria de la Universidad de Córdoba, en relación al efecto de beneficio que tiene el empleo de las herramientas Web 2.0 para el refuerzo de la presencia, la participación y el progreso, como estrategias de desarrollo de los procesos educativos inclusivos.

Entre los objetivos secundarios se encuentra: el uso académico de algunas herramientas Web 2.0, la valoración de las herramientas en relación a las estrategias de presencia, participación y progreso constitutivas del desarrollo de los entornos inclusivos; así como, la relación entre el uso académico y la valoración de la presencia de estos recursos tecnológicos.

En cuanto al diseño de investigación se asienta en un modelo de carácter no experimental y descriptivo, como consecuencia el instrumento planteado ad hoc se realiza a través de escala Likert de cinco opciones, dividido en cuatro bloques diferenciados.

El estudio presenta 55 variables, agrupadas en siete dimensiones, referentes a las herramientas Web 2.0 seleccionadas para esta investigación (redes sociales, YouTube, foros, mensajería instantánea, blog/edublog, wiki, Webquest y procesadores de texto) en función del conocimiento, uso personal, uso académico, valoración de la presencia, de la participación y del progreso de las mismas, por último se ordenan los datos identificativos relativos a elementos socio - demográficos en otra dimensión. Para la construcción del instrumento se tuvo en cuenta la clasificación realizada por Marín en 2012 y adaptado posteriormente por Sampedro y Marín en 2015, adaptándose al área inclusiva.

Para comprobar la validez y la fiabilidad se han realizado dos pruebas, con respecto a esta última, se ha efectuado un enfoque de consistencia interna, a través de la aplicación del parámetro de Alfa de Cronbach cuyo resultado ha sido de 0,928 , lo cual refleja una fiabilidad alta de los ítems expresados en el cuestionario. Por otro lado, en la búsqueda del índice de fiabilidad se ha realizado un análisis de la capacidad de discriminación de los elementos a través de la prueba estadística $\mathrm{t}$ de Student (para muestras independientes) entre las medias de los grupos constituidos, la cual revela un alto poder de discriminación en todos los ítems dado que los valores de significatividad obtenidos son $\mathrm{p}<0,001$, este hecho implica que los elementos congregados en la prueba cumplen con los objetivos planteados para cada una de las cuestiones de la dimensiones.

La muestra participante en el estudio quedó conformada por 215 alumnas y 89 alumnos matriculados en las titulaciones de Grado en Magisterio en Educación Primaria (65 participantes), Educación Física (54), Lengua Extranjera 
(65), Educación Musical (43) y Educación Especial (77) en la Facultad de Ciencias de la Educación de la Universidad de Córdoba y cuya edades estaban comprendidas entre los 22 y los 47 años.

\section{Resultados del estudio}

Los estudiantes participantes en este estudio realizan un uso respecto al ámbito académico alto de los procesadores de textos $(\overline{\mathrm{X}}=4,60)$, de la mensajería instantánea o chat $(\overline{\mathrm{X}}=3,72)$, de YouTube $(\overline{\mathrm{X}}=3,41)$, de los foros $(\overline{\mathrm{X}}=3,39)$, de las redes sociales $(\overline{\mathrm{X}}=3,37)$ y de la wiki $(\overline{\mathrm{X}}=3,16)$, en cambio poseen un menor conocimiento, de los blog y edublogs y de la Webquest ( $\overline{\mathrm{X}}=2,97$ y 2,89 respectivamente).

Asimismo, tratando de establecer diferencias estadísticamente significativas atendiendo al sexo del alumnado, aplicando una prueba t de Student (n.s. $=0.05)$, la cual ha revelado que no existen diferencias significativas respecto a esta variable.

De la misma forma, debido a la adscripción de este grupo de estudiantes a diferentes titulaciones formativas, hemos considerado necesario realizar un Análisis de Varianza (n.s. $=0.05$ ) obteniendo los siguientes resultados:

El alumnado de la titulación de maestro en Educación Especial realiza un mayor uso respecto al ámbito académico de la herramienta Web 2.0, redes sociales que el resto de los discentes de las titulaciones de Educación Física, Lengua Extranjera, Educación Musical y Educación Especial ( $F=8,127$ y p= $<0,001)$;

- Las herramientas Web 2.0 blog y edublogs $(\mathrm{F}=15,370$ y $\mathrm{p}=<0,001)$, foros $(\mathrm{F}=9,070$ y $\mathrm{p}=<0,001)$, Webquest $(\mathrm{F}=8,827$ y $\mathrm{p}=<0,001)$ y wiki $(\mathrm{F}=11,970$ y $\mathrm{p}=<0,001)$ son más utilizadas académicamente por los estudiantes de la titulación de Educación Primaria en comparación con el alumnado de las restantes titulaciones;

- Las herramientas Web 2.0 youtube $(\mathrm{F}=13,400$ y $\mathrm{p}=<0,001)$ y los procesadores de texto $(\mathrm{F}=2,698$ y $\mathrm{p}=0,031)$ son más utilizadas académicamente por el alumnado de la titulación de Educación Musical en comparación con el de las restantes titulaciones;

- El uso respecto al ámbito académico de la mensajería instantánea o chat no es una herramienta Web 2.0 con carácter significativo en función de las titulaciones de los estudiantes participantes en este estudio.

Del mismo modo, se ha procedido a realizar una prueba de comparaciones múltiples post hoc para controlar la tasa de error para el conjunto total de comparaciones (prueba de Scheffé), la cual ha confirmado las diferencias más significativas aquí citadas. 
Por último, hemos considerado analizar si la forma de acceso a Internet es significativa en esta dimensión, por esta razón hemos procedido a realizar un Análisis de Varianza (n.s.=0.05), del cual se han obtenido los siguientes resultados:

- El uso académico de los blogs y edublos y la Webquest es mayor en los alumnos que acceden a Internet a través del móvil en comparación con el resto de estudiantes que realizan otra forma de acceso $(F=2,864$ y $\mathrm{p}=0,015$ );

- El resto de herramientas de este estudio no han reflejado resultados significativos en función del tipo de acceso a Internet para la dimensión uso respecto al ámbito académico de algunas herramientas Web 2.0.

La realización de la prueba de comparaciones múltiples post hoc para controlar la tasa de error para el conjunto total de comparaciones, denominado prueba de Scheffé, ha confirmado las diferencias más significativas que hemos expuesto.

Los resultados en relación a la valoración respecto de la presencia que realiza los participantes de la investigación de las herramientas Web 2.0 reflejan que tienen una mejor valoración de las herramientas Web 2.0, procesadores de textos $(\overline{\mathrm{X}}=4,18)$, redes sociales $(\overline{\mathrm{X}}=3,73)$ y mensajería instantánea $(\overline{\mathrm{X}}=3,54)$ para reforzar la presencia como estrategia que desarrolla procesos educativos inclusivos. En cambio, valora en menor medida la herramienta YouTube $(\overline{\mathrm{X}}=3,46)$, los foros $(\overline{\mathrm{X}}=3,40), \operatorname{los}$ blog y edublogs $(\overline{\mathrm{X}}=3,35)$, la wiki $(\overline{\mathrm{X}}=3,08)$ y la Webquest $(\overline{\mathrm{X}}=3,03)$.

En un segundo momento, hemos tratado de establecer diferencias estadísticamente significativas atendiendo al sexo del alumnado, aplicando una prueba t de Student (n.s.=0.05) cuyos resultados han mostrado que no existe diferencias significativas respecto a esta variable de diferenciación.

Asimismo, debido a la procedencia dispar de este grupo de estudiantes en referencia a su titulación formativa, hemos realizado un Análisis de Varianza (n.s. $=0.05)$ obteniendo los siguientes valores representativos:

- El alumnado de las titulaciones de maestro en Educación Primaria y Lengua Extranjera realizan una valoración más favorable de la herramienta Web 2.0, redes sociales como refuerzo de la presencia en los entornos inclusivos, que el resto de los alumnos de las titulaciones de Educación Física, Educación Musical y Educación Especial ( $\mathrm{F}=3,151$ y p=0,015);

- Las herramientas Web 2.0, blog y edublogs, es valorada más favorablemente por los estudiantes de la titulación de Lengua Extranjera, en entornos inclusivos atendiendo a la presencia, en comparación con el alumnado de las restantes titulaciones $(\mathrm{F}=10,155$ y $\mathrm{p}=<0,001)$. 
- Los discentes procedentes de la titulación de Educación Primaria valoran más positivamente los foros $(\mathrm{F}=6,975 \mathrm{y} \mathrm{p}=<0,001)$, la mensajería instantánea $(\mathrm{F}=6,328$ y $\mathrm{p}=<0,001)$, la Webquest $(\mathrm{F}=2,931$ y $\mathrm{p}=0,021)$, la wiki $(\mathrm{F}=5,016$ y $\mathrm{p}=0,001)$ en los entornos inclusivos atendiendo a la presencia en comparación con el alumnado de las otras titulaciones.

- $\quad$ La herramienta Web 2.0, youtube, es más valorada como refuerzo de la presencia en los entornos inclusivos, por los alumnos de la titulación de Educación Especial en comparación con los estudiantes de las restantes titulaciones $(\mathrm{F}=5,424$ y $\mathrm{p}=<0,001)$;

- La valoración de los procesadores de texto en entornos inclusivos atendiendo a la presencia no es una herramienta Web 2.0 con carácter significativo en función de las titulaciones del alumnado participante en este estudio.

La prueba de Scheffé sobre comparaciones múltiples post hoc para controlar la tasa de error para el conjunto total de comparaciones, ha validado las diferencias más significativas que hemos expuesto.

Por último, hemos considerado analizar si la forma de acceso a Internet es significativa en esta dimensión, por esta razón hemos procedido a realizar un Análisis de Varianza (n.s.=0.05), el cual ha revelado que esta variable no es significativa para la dimensión valoración de algunas herramientas Web 2.0 en entornos inclusivos atendiendo a la presencia.

Respecto a la valoración de la participación en los entornos inclusivos que realiza el alumnado de las herramientas Web 2.0 los resultados muestran que los estudiantes participantes en este estudio valoran más positivamente los procesadores de textos $(\overline{\mathrm{X}}=4,19)$, las redes sociales $(\overline{\mathrm{X}}=3,88)$, YouTube $(\overline{\mathrm{X}}=3,60)$, la mensajería instantánea $(\overline{\mathrm{X}}=3,67)$ y los foros $(\overline{\mathrm{X}}=3,52)$ como herramientas Web 2.0 que refuerzan la participación, estrategia de desarrollo de los procesos educativos inclusivos. En cambio realizan una menor valoración de los blog y edublogs, de la wiki y de la Webquest ( $\overline{\mathrm{X}}=3,40 ; 3,21$ y 3,05 respectivamente).

Considerando esta misma dimensión "Valoración de algunas herramientas Web 2.0 en entornos inclusivos atendiendo a la participación" hemos tratado de establecer diferencias estadísticamente significativas atendiendo al sexo del alumnado, aplicando una prueba t de Student (n.s. $=0.05)$, la cual ha revelado los siguientes resultados más significativos:

- Las redes sociales $(\mathrm{t}=-2,830$ y $\mathrm{p}=0,003)$ y la mensajería instantánea $(\mathrm{t}=-3,293$ y $\mathrm{p}=0,001)$ son mejor valoradas como herramientas Web 2.0 que refuerzan la participación en entornos inclusivos por las mujeres que por los hombres; 
- El resto de las herramientas Web 2.0 de este estudio no revelan diferencias estadísticamente significativas en relación al sexo para esta dimensión.

De la misma forma, debido a la adscripción de este grupo de estudiantes a diferentes titulaciones formativas, hemos considerado necesario realizar un Análisis de Varianza (n.s. $=0.05$ ) obteniendo los siguientes valores representativos:

- Los estudiantes de la titulación de maestro en Lengua Extranjera realizan una valoración más favorable de las herramientas Web 2.0, redes sociales $(\mathrm{F}=4,434$ y $\mathrm{p}=0,002)$, blog y edublogs $(\mathrm{F}=11,347$ y $\mathrm{p}=<0,001)$, mensajería instantánea $(\mathrm{F}=7,983$ y $\mathrm{p}=<0,001)$ y youtube $(\mathrm{F}=5,895$ y $\mathrm{p}=<0,001)$ como refuerzo de la participación en los entornos inclusivos, que el resto de los alumnos de las titulaciones de Educación Primaria, Educación Física, Educación Musical y Educación Especial;

- El alumnado procedente de la titulación de Educación Primaria valoran más positivamente los foros $(\mathrm{F}=7,785 \mathrm{y} \mathrm{p}=<0,001)$, la Webquest $(\mathrm{F}=4,557$ y $\mathrm{p}=0,001)$ y la wiki $(\mathrm{F}=9,464$ y $\mathrm{p}=<0,001)$ en entornos inclusivos atendiendo a la participación en comparación con el resto de estudiantes de las otras titulaciones;

- La valoración de los procesadores de texto en entornos inclusivos atendiendo a la participación no es una herramienta Web 2.0 con carácter significativo en función de las titulaciones del alumnado participante en este estudio.

Además, se ha procedido a realizar una prueba de comparaciones múltiples post hoc para controlar la tasa de error para el conjunto total de comparaciones, denominado prueba de Scheffé, la cual ha confirmado las diferencias más significativas que hemos expuesto.

Por último, hemos considerado analizar si la forma de acceso a Internet es significativa en esta dimensión, por esta razón hemos procedido a realizar un Análisis de Varianza (n.s.=0.05), el cual ha revelado:

- La valoración de las redes sociales $(\mathrm{F}=2,456$ y $\mathrm{p}=0,034)$ y los procesadores de textos $(\mathrm{F}=2,441 \mathrm{y} \mathrm{p}=0,034)$ como herramientas Web 2.0 que refuerzan la participación en los procesos educativos inclusivos es más favorable en los alumnos que acceden a Internet a través del móvil en comparación con el resto de estudiantes que realizan otra forma de acceso;

- Los discentes que acceden a Internet a través de todas las modalidades de forma conjunta valoran más positivamente la mensajería ins- 
tantánea en los entornos inclusivos para reforzar la participación $(\mathrm{F}=2,268$ y $\mathrm{p}=0,048)$;

- $\quad$ El resto de herramientas de este estudio no han reflejado resultados significativos en función del tipo de acceso a Internet para la dimensión valoración de algunas herramientas Web 2.0 en entornos inclusivos atendiendo a la participación.

La prueba de Scheffé sobre comparaciones múltiples post hoc para controlar la tasa de error para el conjunto total de comparaciones, ha validado las diferencias más significativas que hemos expuesto.

Los alumnos participantes en este estudio realizan una mejor valoración de los procesadores de textos $(\overline{\mathrm{X}}=4,27)$, de las redes sociales $(\overline{\mathrm{X}}=3,79)$, de la mensajería instantánea $(\overline{\mathrm{X}}=3,61)$, de YouTube $(\overline{\mathrm{X}}=3,60)$, de los foros $(\overline{\mathrm{X}}=3,55)$ y de los blog y edublogs $(\overline{\mathrm{X}}=3,53)$ como herramientas Web 2.0 que refuerzan el progreso en los entornos inclusivos, en cambio poseen una menor valoración de la wiki $(\overline{\mathrm{X}}=3,34)$ y de la Webquest $(\overline{\mathrm{X}}=3,21)$ a este respecto.

En este sentido, en relación a la dimensión "Valoración de algunas herramientas Web 2.0 en entornos inclusivos atendiendo al progreso" hemos tratado de establecer diferencias estadísticamente significativas atendiendo al sexo del alumnado, aplicando una prueba t de Student (n.s.=0.05) la cual revela que solamente los foros $(\mathrm{t}=-3,261$ y $\mathrm{p}=0,001)$ son mejor valorados por las mujeres que refuerzan el progreso para desarrollar procesos educativos inclusivos, en comparación con los hombres.

Asimismo, debido a la procedencia dispar de este grupo de estudiantes en referencia a su titulación formativa, hemos realizado un Análisis de Varianza (n.s.=0.05) obteniendo los siguientes valores representativos:

- Los estudiantes de la titulación de maestro en Educación Primaria realizan una valoración más favorable de las herramientas Web 2.0, redes sociales $(\mathrm{F}=3,857$ y $\mathrm{p}=0,005)$, blog y edublogs, $(\mathrm{F}=7,789$ y $\mathrm{p}=<0,001)$, los foros $(\mathrm{F}=7,352$ y $\mathrm{p}=<0,001)$, mensajería instantánea $(\mathrm{F}=6,664$ y $\mathrm{p}=<0,001)$, la Webquest $(\mathrm{F}=3,920$ y $\mathrm{p}=0,004)$, wiki $(\mathrm{F}=5,625$ y $\mathrm{p}=<0,001)$ y youtube $(\mathrm{F}=3,671$ y $\mathrm{p}=0,006)$ como refuerzo del progreso en los entornos inclusivos, que el resto del alumnado de las titulaciones de Educación Primaria, Educación Física, Educación Musical y Educación Especial;

- La valoración de los procesadores de texto en entornos inclusivos atendiendo al progreso no es una herramienta Web 2.0 con carácter significativo en función de las titulaciones del alumnado participantes en este estudio. 
Además, se ha procedido a realizar una prueba de comparaciones múltiples post hoc para controlar la tasa de error para el conjunto total de comparaciones, denominado prueba de Scheffé, la cual ha confirmado las diferencias más significativas que hemos expuesto.

Finalmente, hemos considerado analizar si la forma de acceso a Internet es significativa en la dimensión "Valoración de algunas herramientas Web 2.0 en entornos inclusivos atendiendo al progreso", consecuentemente hemos procedido a realizar un Análisis de Varianza (n.s. $=0.05$ ), el cual ha revelado:

- La valoración de la wiki $(\mathrm{F}=3,499$ y $\mathrm{p}=0,004)$ y YouTube $(\mathrm{F}=2,660$ y $\mathrm{p}=0,023)$ como herramientas Web 2.0 que refuerzan el progreso en los procesos educativos inclusivos es más favorable en los alumnos que acceden a Internet a través del móvil en comparación con el resto de estudiantes que realizan otra forma de acceso;

- Los procesadores de textos son valorados principalmente, como herramienta Web 2.0 que refuerza el progreso en entornos inclusivos, por los estudiantes que acceden a Internet a través de todas las modalidades de forma conjunta $(\mathrm{F}=5,134$ y $\mathrm{p}=<0,001)$;

- El resto de herramientas de este estudio no han reflejado resultados significativos en función del tipo de acceso a Internet para la dimensión valoración de algunas herramientas Web 2.0 en entornos inclusivos atendiendo al progreso.

Para controlar la tasa de error para el conjunto total de comparaciones, prueba de Scheffé, de comparaciones múltiples post hoc, ha revelado las diferencias más significativas que hemos expuesto.

\section{Discusión y consideraciones finales}

Los estudiantes utilizan mayoritariamente los procesadores de texto, la mensajería instantánea, YouTube, los foros, las redes sociales y la wiki, las cuales son utilizadas académicamente en igual proporción por ambos sexos (BOZA; CONDE, 2015). Las herramientas Web 2.0 menos usadas académicamente son las Webquest y los blog y edublogs a diferencia de los resultados alcanzados en los trabajos de Marín (2016) y Molina, Valenciano y Valencia-Peris (2015).

Por este motivo, el uso académico de las herramientas Web 2.0 se ve condicionado por las titulaciones en las que se inscribe el alumnado a excepción de la mensajería instantánea, en este sentido, los blog y edublogs, los foros, la Webquest y la wiki son mayormente utilizadas en al ámbito académico por los estudiantes de Educación Primaria (MARÍN; MUÑOZ; SAMPEDRO, 2014), 
las redes sociales por Educación Especial y, YouTube y los procesadores de texto por el alumnado de Educación Musical.

Además, el tipo de acceso a Internet también es un condicionante para el uso académico de las herramientas Web 2.0, por ejemplo, las herramientas sobre todo utilizadas académicamente a través del acceso por móvil son los blog y edublogs y la Webquest, en cambio el uso académico de las restantes herramientas Web 2.0 no se ve afectado por el tipo de acceso a Internet (BOZA; CONDE, 2015). Atendiendo a la clasificación de Castaño et al. (2008), los estudiantes usan mayoritariamente de manera académica las herramientas de publicación si el acceso lo realizan a través del móvil, en cambio, las herramientas Web 2.0 que se clasifican en las tipologías de las aplicaciones on-line, la gestión de la información y los canales de comunicación no se ven afectadas por el tipo de acceso (MARÍN, 2012; SAMPEDRO; MARÍN, 2015).

Se manifiesta que los procesadores de texto, las redes sociales y la mensajería instantánea son las herramientas Web 2.0 mejor valoradas por los estudiantes para reforzar la presencia en los entornos inclusivos como reflejan Pegalajar y Colmenero (2013), es decir, que según Castaño et al. (2008) y Lozano, Castillo y Veas (2014) los canales de información y algunas aplicaciones on-line serían las que mejor fomentan la presencia como estrategia de desarrollo en los entornos inclusivos frente a otras aplicaciones on-line y herramientas de gestión de la información como los foros, YouTube, los blog y edublogs, la wiki y la Webquest que la refuerzan menos, pues como señalan Leiva y Gómez (2017), la inclusión es un proceso continuo que trata de favorecer la presencia y la, participación de los alumnos, de ahí que llamé la atención que estos últimos recursos digitales que tienen un alto calado entre los alumnos tengan esa baja presencia.

Todas las herramientas Web 2.0, a excepción de los procesadores de texto, están condicionadas en su valoración de la presencia respecto a la titulación del alumnado que participa en este estudio. De esta manera, los estudiantes de Lengua Extranjera valoran más positivamente las redes sociales y los blog y edublogs, YouTube por Educación Especial y, los foros, la mensajería instantánea, la Webquest y la wiki por Educación Primaria, como herramientas Web 2.0 que refuerzan la presencia en los entornos inclusivos. En este sentido y tal como sugería Arnaiz (2003, p. 167) las herramientas Web 2.0 es un catalizador para incluir a todos los estudiantes, además, de para ayudar en el acceso de todos y todas al currículum, opinión que hoy sustentan autores como Yu et al. (2016) o Peñafiel (2012).

La valoración de las herramientas Web 2.0 atendiendo a la participación en los entornos inclusivos, en este sentido y tal como apuntan Cabero y Córdoba (2009, p. 73) una de las posibilidades y ventajas de las herramientas Web 2.0 en el ámbito de la diversidad y la inclusión es que favorecen el desarrollo de la autonomía y la iniciativa propiciando la implicación en los entornos educativos, los alumnos que participan en este estudio valoran positivamente los procesadores de texto, las redes sociales, YouTube, la mensajería instantánea y los foros como herramientas que refuerzan la participación en los entornos inclusivos como reflejan Toledo 
(2013), Lozano, Castillo y Veas (2014) y Feng y Xie (2015), en cambio los blog y edublogs la wiki y la Webquest no son también valoradas en este sentido a diferencia de los datos aportados por Marín (2013) y Troncoso, Martínez y Raposo (2014), quienes señalan la gran utilidad de esta herramientas para la inclusión educativa.

$\mathrm{Al}$ igual que ocurría con la valoración de la presencia, todas las herramientas Web 2.0, a excepción de los procesadores de texto (BOZA; CONDE, 2015), están condicionadas en su valoración de la participación respecto a la titulación del alumnado que participa en este estudio. De esta manera, los estudiantes de Lengua Extranjera valoran más positivamente las redes sociales y los blog y edublogs, la mensajería instantánea y YouTube, en cambio, los foros, la Webquest y la wiki lo son por Educación Primaria, como herramientas Web 2.0 que fomentan la participación en los entornos inclusivos, datos en general que no coinciden con el estudio de Cabero, Fernández y Barroso (2016), quienes indican que las titulaciones de Educación Musical y Especial son más proclives a tener una visión positiva y no las de Lengua Extranjera y Primaria, como sucede en este caso.

El tipo de acceso a Internet es también un condicionante en la valoración del efecto de beneficioso de algunas herramientas Web 2.0 para la participación como estrategia que desarrolla los entornos inclusivos. En este sentido, las redes sociales y los procesadores de texto son mejor valorados (BARRAGÁN; RUíZ, 2013), si el acceso se realiza a través del móvil, en cambio, la mensajería instantánea se valora más positivamente si el tipo de acceso es conjunto de todas las herramientas. El resto de herramientas Web 2.0 (blog y edublogs, foros, Webquest, wiki y YouTube) no se ven afectadas por el acceso en lo relativo a la valoración de la participación (MARÍN; SAMPEDRO, 2016), considerando la clasificación de Castaño et al. (2008), los alumnos usan mayoritariamente de manera personal las herramientas de las aplicaciones on-line, la gestión de la información y los canales de comunicación y menos la de publicación de contenidos (HUANG; HOOD; YOO, 2013).

Los estudiantes que participan en este estudio valoran positivamente los procesadores de texto, las redes sociales, la mensajería instantánea, YouTube y los foros como herramientas que refuerzan el progreso en los entornos inclusivos (BOZA; CONDE, 2015; CABERO; FERNÁNDEZ; BARROSO, 2016). Los blog y edublogs la wiki y la Webquest no son también valoradas en este sentido, tal y como ocurría con la participación a diferencia de los resultados alcanzados por Marín (2012).

De la misma forma que ocurría con la valoración de la presencia y la participación, todas las herramientas Web 2.0, a excepción de los procesadores de texto, están condicionadas en su valoración del progreso respecto a la titulación del alumnado que participa en este estudio. En este sentido, los estudiantes de Educación Primaria son los que valoran más positivamente las redes sociales y los blog y edublogs, la mensajería instantánea, YouTube, los foros, la Webquest y la wiki, como herramientas Web 2.0 que fomentan el progreso en los entornos inclusivos (MARÍN, 2013; MARÍN; SAMPEDRO, 2016). 
Las redes sociales, los blog y edublogs, los foros, la mensajería instantánea, la Webquest y la wiki se valoran igual sea cual sea el tipo de acceso en función del progreso, por el contrario, la valoración sobre el efecto más beneficioso de las herramientas Web 2.0 en relación al progreso como herramienta que desarrolla los entornos inclusivos susceptible del tipo de acceso a Internet son de las herramientas procesadores de texto a través del acceso conjunto de todas las modalidades, $\mathrm{y}$, la wiki y YouTube por los estudiantes que acceden a Internet a través del móvil.

La relación entre el uso académico de algunas herramientas Web $2.0 \mathrm{y}$ la valoración en el empleo de las mismas para reforzar la presencia, la participación y el progreso como estrategias para el desarrollo en los entornos inclusivos, el estudio revela que si existe relación entre estas dos variables, por otro, el uso de las herramientas Web 2.0, favorecen y propician las características esenciales de los entornos inclusivos: presencia (dado que ayudan al acceso de todas las personas), participación (ya que posibilitan la autonomía y la toma de decisiones) y progreso (porque permiten la oportunidad de lograr los conocimientos) al igual que en el trabajo llevado a cabo por Cabero, Fernández y Barroso (2016).

En este sentido, en relación, al uso académico y la valoración de la presencia como estrategia que desarrolla los procesos inclusivos, el estudio ha reflejado que los alumnos que realizan un uso académico de alguna de las herramientas Web 2.0 valoran a la misma en relación a la presencia (CABERO; FERNÁNDEZ; BARROSO, 2016). Por otro lado, los estudiantes que utilizan de forma académica el YouTube valoran en relación a la presencia en los entornos inclusivos todas las herramientas Web 2.0 de este estudio (redes sociales, blog y edublogs, foros, mensajería instantánea, Webquest, wiki, YouTube y procesadores de texto), por el contrario, los alumnos que usan académicamente los procesadores de texto sólo valoran éstos como herramienta que refuerza la presencia en los entornos inclusivos.

Respecto a la relación entre el uso académico y la valoración de la participación en los entornos inclusivos de las herramientas Web 2.0, se manifiesta que los estudiantes que usan académicamente alguna de las herramientas Web 2.0 realizan una valoración atendiendo a la participación en los entornos inclusivos de la misma; así mismo, quien realiza un uso académico de los foros, de la wiki y de YouTube valora positivamente todas las herramientas (CABERO; FERNÁNDEZ; BARROSO, 2016), en cambio sí sólo usan los procesadores de texto sólo valoran estos.

Considerando la relación entre el uso académico y la valoración del progreso en los entornos inclusivos de algunas herramientas Web 2.0, el estudio revela que los estudiantes que usan académicamente alguna de las herramientas Web 2.0 realizan una valoración atendiendo al progreso en los entornos inclusivos de la misma; así mismo, quien realiza un uso académico de los foros y de YouTube valora las herramientas mismas herramientas (BOZA; CONDE, 2015), en cambio sí sólo usan los procesadores de texto sólo valoran estos. 
Por tanto, mientras que se utilice académicamente alguna herramienta esta misma es valorada respecto a la presencia, participación y progreso en los entornos inclusivos, además, quien utiliza los procesadores de texto de forma académica sólo aprecian esta herramienta para la presencia, la participación y el progreso en los entornos inclusivos, en cambio en las tres estrategias de desarrollo de los entornos inclusivos (presencia, participación y progreso) la utilización académica de YouTube favorece que se valoren la mayoría de las herramientas.

\section{Referencias}

ARAUJO, M.E. Formación del Docente de Educación Media General desde el Enfoque por Competencias. Scientific, v. 1, n. 2, p. 181-199, 2016.

ARNAIZ, P. Educación inclusiva: una escuela para todos. Málaga: Aljibe, 2003.

BARRAGÁN, R.; RUIZ, E. Brecha de género e inclusión digital. El potencial de las redes sociales en educación. Profesorado, Granada, v. 17, n. 1, p. 309-323, 2013.

BOZA, A.; CONDE, S. Web 2.0 en educación superior: formación, actitud, uso, impacto, dificultades y herramientas. Digital Education Review, Barcelona, n. 28, p. 45-58, 2015.

CABERO, J. Educación 2.0. ¿¿Marca, moda o nueva visión de la educación? In: CASTAÑO, C. (Coord.). Web 2.0: El uso de la web en la sociedad del conocimiento. Investigación e implicaciones educativas. Venezuela: Universidad Metropolitana, 2009. p. 13-34.

CABERO, J.; CÓRDOBA, M. Inclusión Educativa: inclusión digital. Revista de Educación Inclusiva, Jaén, v. 2, n. 1, p. 61-78, 2009.

CABERO, J.; FERNÁNDEZ, J.M.; BARROSO, J. Los alumnos del grado de Magisterio: TIC y discapacidad. Revista Electrónica de Investigación Educativa, Murcia, v. 18, n. 3, p. 106-120, 2016.

CARRASCO, S.; BALDIVIESO, S.; DI LORENZO, L. Formación en investigación educativa en la sociedad digital. Una experiencia innovadora de enseñanza en el nivel superior en el contexto latinoamericano. Revista de Educación a Distancia, Murcia, v. 48, 2016.

CASTAÑO, C.; MAIZ, I.; PALACIO, G.V.; ILLAROEL, J.D. Prácticas educativas en entornos web 2.0. Madrid: Síntesis, 2008.

COZAR-GUTIÉRREZ, R.; SÁEZ-LÓPEZ, J.M. Realidad aumentada, proyectos en el aula de primaria: experiencias y casos en Ciencias Sociales. Revista de Educación Mediática y TIC, Córdoba, v. 6, n. 1, p. 165-180, 2017.

DABBAGH, N.; FAKE, H. College Students' Perceptions of Personal Learning Environments Through the Lens of Digital Tools, Processes and Spaces. Journal of New Approaches in Educational Research, Londres, v. 6, n. 1, p. 28-36, 2017.

DE LA HORRA, G.I. Realidad Aumentada: Una revolución educativa. EDMETIC Revista de Educación Mediática y TIC, v. 6, n. 1, p. 9-22, 2017. 
DE LA TORRE, A. Web Educativa 2.0. EDUTEC, Islas Baleares, n. 20, 2006. Disponible en: <http://www.edutec.es/revista/index.php/edutec-e/article/view/515/248 >. Acceso en: 23 feb. 2013.

FENG, Y.; XIE, W. Digital Divide 2.0: The Role of Social Networking Sites in Seeking Health Information Online From a Longitudinal Perspective. Journal of Health Communication, Holand, v. 20, n. 1, p. 60-68, 2015.

GALLEGO, J.L.; RODRÍGUEZ, A. Tendencias en la formación inicial del profesorado de educación especial. Revista Iberoamericana sobre Calidad, Eficacia y Cambio en Educación, Madrid, v. 5, n. 3, p. 102-117, 2007.

GRIEVE, R.; PADGETT, C.R.; MOFFITT, R.L. Assignments 2.0: the role of social presence and computer attitudes in student preferences for online versus offline marking. Internet and Higher Education, London, v. 28, p. 8-16, 2015.

HUANG, W.; HOOD, D.W.; YOO, S.J. Gender divide and acceptance of collaborative web 2.0 applications for learning in higher education. Internet and Higher Education, London, v. 16, p. 57-65, 2013.

LEIVA, J.J.; GÓMEZ, M.Á. La educación inclusiva como constructo pedagógico en el alumnado universitario de educación primaria. Revista de Educación Inclusiva, Jaén, v. 8, n. 2, p. 185-200, 2017.

LOZANO, J.; CASTILLO, I-S.; VERAS, A. Alumnado con necesidades específicas de apoyo educativo de Educación Secundaria Obligatoria y el consumo de las TIC en la región de Murcia.Etc@net, v. 14, n. 1, p. 57-67, 2014.

MARÍN, V. (Coord.). Diseño e implementación de una metodología de enseñanza a través de la plataforma Moodle, como herramienta para el desarrollo de la formación del alumnado de Grado de Primaria. Sevilla: SAV, 2012.

El Blog: pensamiento de los profesores en formación en Educación Primaria. Opción, Venezuela, año 32, n. 79, p. 145-162, 2016.

. Innovando en las aulas universitarias a través de los blogs desde una perspectiva inclusiva. Interaç̧óes, Lisboa, v. 9, n. 23, p. 88-101, 2013.

MARÍN, V.; MUÑOZ, J.M.; SAMPEDRO, B.E. Los blogs educativos como herramienta para trabajar la inclusión desde la educación superior. Ensayos, Revista de la Facultad de Educación de Albacete, v. 29, n. 2, p. 115-127, 2014.

MARÍN, V:; SAMPEDRO, B.E. Web 2.0 en la dinamización y participación familiar y comunitaria. Journal of New Approaches in Educational Research, Alicante, v. 5, n. 1, p. 38-43, 2016.

MARIÑO, S.I.; ALFONZO, P.L.; CAVALIERI, J.I. Un taller en herramientas web para la sistematización de datos. Una experiencia de actualización de conocimientos. EDUTEC, Islas Baleares, n. 50, 2014.

MIRABAL, A.R.; GÓMEZ, M.G.; GONZÁLEZ, L.A. Uso de la plataforma Moodle como apoyo a la docencia presencial universitaria. EDMETIC, Córdoba, v. 4, n. 1, p. 133-155, 2015. 
MOLINA, P.; VALENCIANO, J.; VALENCIA-PERIS, A. Los blogs como entornos virtuales de enseñanza y aprendizaje en Educación Superior. Revista Complutense de Educación, Madrid, v. 26, p. 15-38, 2015.

ORGANIZACIÓN DE LAS NACIONES UNIDAS PARA LA EDUCACIÓN, LA CIENCIA Y LA CULTURA (UNESCO). Conferencia Mundial de Educación Superior: las nuevas dinámicas de la educación superior y de la investigación para el cambio social y el desarrollo. Paris: UNESCO, 2009.

48. a Conferencia Internacional de Educación: la educación inclusiva: el camino hacia el futuro. Ginebra: UNESCO, 2008a.

. Estándares de competencias en TIC para docentes. Paris: UNESCO, $2008 \mathrm{~b}$.

PEGALAJAR, M.C.; COLMENERO, M.J. PICAA: Aplicación móvil de aprendizaje para la inclusión educativa del alumnado con discapacidad. Etc@net, v. 1, n. 13, p. 94-146, 2013.

PENAAFIEL, F. Educación inclusiva y era digital. Un nuevo planteamiento de actuación. Etic@net, v. 12, n. 2, p. 168-186, 2012.

ROBLIZO, M.; COZAR, R. Usos y competencias en TIC en los futuros maestros de educación infantil y primaria: hacia una alfabetización tecnológica real para docentes. Pixel-Bit Revista de Medios y Educación, Sevilla, v. 47, p. 23-39, 2015.

SALES, A. La formación inicial del profesorado ante la diversidad: una propuesta metodológica para el nuevo espacio europeo de educación superior. Revista Interuniversitaria de Formación del Profesorado, Murcia, v. 20, n. 3, p. 201-217, 2006.

SAMPEDRO, B.E.; MARÍN, V. Conocimiento de los futuros educadores sociales de las herramientas Web 2.0. Pixel Bit Revista de Medios y Educación, Sevilla, v. 47, p. 41-58, 2015.

TOLEDO, P. Las tecnologías de la información y la comunicación y la inclusión educativa. In: BARROSO; J.; CABERO, J. Nuevos escenarios digitales. Las tecnologías de la información y la comunicación aplicadas a la formación y el aprendizaje curricular. Madrid: Pirámide, 2013. p. 411-426.

TRONCOSO, A.B.; MARTÍNEZ, M.E.; RAPOSO, M. La inclusión del alumno con discapacidad intelectual a partir del uso de blogs: una experiencia educativa. Revista Latinoamericana de Educación Inclusiva, Chile, v. 7, n. 2, p. 195-211, 2014.

YU, B.; NDUMU, A.; LIU, J.; FEN, Z. E-inclusion or digital divide: on integration model of digital inequality. Proceeding of Association for Information Science and Technology, v. 53, n. 1, p. 1-5, 2016.

Recibido en 05 de junio de 2016.

Acepto en 23 de junio de 2017. 